\title{
Radiative efficiency of hot accretion flow and the radio/X-ray correlation in $\mathrm{X}$-ray binaries
}

Fu-Guo Xie

Key Laboratory for Research in Galaxies and Cosmology, Shanghai Astronomical Observatory, Chinese Academy of Sciences, 80 Nandan Road, Shanghai 200030, China

email: fgxie@shao.ac.cn

\begin{abstract}
Significant progresses have been made since the discovery of hot accretion flow, a theory successfully applied to the low-luminosity active galactic nuclei (LLAGNs) and black hole (BH) X-ray binaries (BHBs) in their hard states. Motivated by these updates, we reinvestigate the radiative efficiency of hot accretion flow. We find that, the brightest regime of hot accretion flow shows a distinctive property, i.e. it has a constant efficiency independent of accretion rates, similar to the standard thin disk. For less bright regime, the efficiency has a steep positive correlation with the accretion rate, while for faint regime typical of advection-dominated accretion flow, the correlation is shadower. This result can naturally explain the observed two distinctive correlations between radio and X-ray luminosities in black hole X-ray binaries. The key difference in systems with distinctive correlations could be the viscous parameter, which determines the critical luminosity of different accretion modes.
\end{abstract}

Keywords. accretion, accretion discs - black hole physics - X-ray: binaries

\section{Introduction}

The accretion flow around compact objects like black holes can emit a significant amount of energy out. One way to characterise this significance is through radiative efficiency, a ratio between the radiative power $L$ to the rest-mass energy, i.e. $\epsilon \equiv L / \dot{M} c^{2}$, where $\dot{M}$ is the mass accretion rate of the system. Depending on their temperature, the accretion flows are categorized into hot and cold series. The radiative efficiency is fairly clear for the cold ones, i.e. it is nearly a constant with typical value $10 \%$ for the standard Shakura-Sunyaev disk (Shakura \& Sunyaev (1973), hereafter SSD), and slightly lower for the Slim disk (Abramowicz et al. (1988)) due to energy advection.

Two important progresses have been achieved in the hot accretion theory (see Xie \& Yuan (2012) and Yuan \& Narayan (2014) for evidences.), a theory that has been successfully applied in the LLAGNs and the hard states of BHBs, see Yuan \& Narayan (2014). First, a large fraction of the accreting material will not be accreted into the BH, but rather be repelled out in the form of outflow (Yuan et al. (2012a), Yuan et al. (2012b)). Secondly, the turbulent dissipation can heat the electrons directly, which leads to an enhancement in the radiative efficiency. Hot accretion flow include both the advectiondominated accretion flow (ADAFs; Narayan \& Yi (1994)) at low luminosities and the luminous hot accretion flow (LHAF; Yuan (2001)) at high luminosities. At even higher accretion rates, thermal instability leads the hot accretion flow to a clumpy configuration, i.e. numerous cold clumps embedded within the hot gas. Eventually it will be a disccorona structure for bright AGNs and BHBs in soft states (e.g. Yang et al. (2015)). 

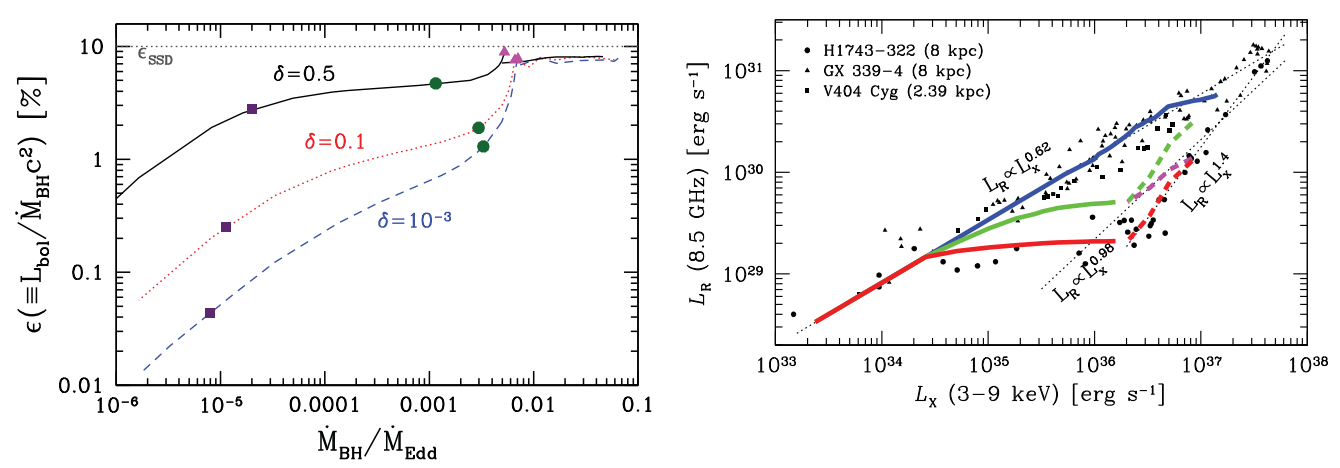

Figure 1. Left Radiative efficiency of hot accretion flows. Parameter $\delta$ indicates the fraction of viscous heating that directly heats the electrons. The green filled circles separate different modes of hot accretion flows. Adapted from Xie \& Yuan (2012). Right Radio/X-ray luminosity relationship in black hole X-ray binaries. The red and green curves are for $\alpha=0.06$, while the blue curves are for $\alpha=0.6$.

\section{Radiative efficiency and radio/X-ray correlation}

In ADAFs, most of the viscously liberated energy will be stored as the gas internal energy and advected into BH. Consequently the efficiency is relatively low, which positively correlates with the accretion rate. Besides, more faction of direct viscous heating to the electrons will lead to higher radiative efficiency. The moderate luminosity (LHAF) regime has a very steep $\epsilon-\dot{M}$ correlation. Finally, at the brightest regime where the accretion flow is two-phase, the efficiency is nearly a constant, similar to that of SSD. These results are shown in the left panel of Fig. 1.

One application of the radiative efficiency is to understand the radio/X-ray luminosity correlation observed in black hole X-ray binaries. In X-ray binaries, both the conventional $p \sim 0.6$ (Corbel et al. (2013); in the form $L_{\mathrm{R}} \propto L_{\mathrm{X}}^{p}$, where $L_{\mathrm{R}}$ and $L_{\mathrm{X}}$ are respectively the radio and the X-ray luminosities.) and the new hybrid (Coriat et al. (2011)) correlations are observed (cf. right panel of Fig. 1, Yuan \& Xie (in preparation).).

In the hot accretion flow - jet model, the radio emission comes from the jet, while the $\mathrm{X}$-ray emission comes from the hot accretion flow. With a fixed function of mass ejection rate into the jet versus the accretion rate, we can reproduce the $p \sim 0.6$ correlation at low luminosities. Because the enhancement of efficiency for the bright ADAF and LHAF branches, the X-ray luminosity increases much faster than the radio luminosity in this regime, leads to a much flatter radio/X-ray correlation. At the bright two-phase accretion flow branch, the efficiency is nearly a constant, which reproduces the $p \sim 1.3$ correlation.

\section{References}

Abramowicz, M. A., Czerny B., Lasota, J. P., \& Szuszkiewicz, E. 1988, ApJ, 332, 646

Corbel, S., et al. 2013, MNRAS, 428, 2500

Coriat, M., et al. 2011, MNRAS, 414, 677

Narayan, R. \& Yi, I. 1994, ApJ, 428, L13

Shakura, N. I. \& Sunyaev, R. A. 1973, A\&A, 24, 337

Xie, F. G. \& Yuan, F., 2012, MNRAS, 427, 1580

Yang, Q. X., Xie, F. G., Yuan, F., Zdziarsksi, A. A., Ho, L. C., \& Yu, Z., 2015, MNRAS, 447, 1692

Yuan, F. 2001, MNRAS, 324, 119

Yuan, F., Bu, D., \& Wu, M. 2012b, ApJ, 761, 130

Yuan, F., Wu, M., \& Bu, D. 2012a, ApJ, 761, 129

Yuan, F. \& Narayan, R. 2014, Ann. Rev. Astron. Astroph., 52, 529 